\title{
Local Inflammatory Response after Topically Applied Burn Stimuli Investigated in Excised Human Skin
}

Katrin Tiffner ${ }^{1}$, Martin Funk ${ }^{3}$, Lars Kamolz ${ }^{2}$, Thomas Birngruber ${ }^{1}$

CONTACT

JOANNEUM RESEARCH Forschungsgesellschaft mbH

HEALTH

Institute for Biomedicine and Health Sciences

Dr Thomas Birngruber

Neue Stiftingtalstrasse 2
8010 Graz

Phone +43 316 876-4114

omas.birngruber@joanneum.at

health@joanneum.at

www joanneum at/health

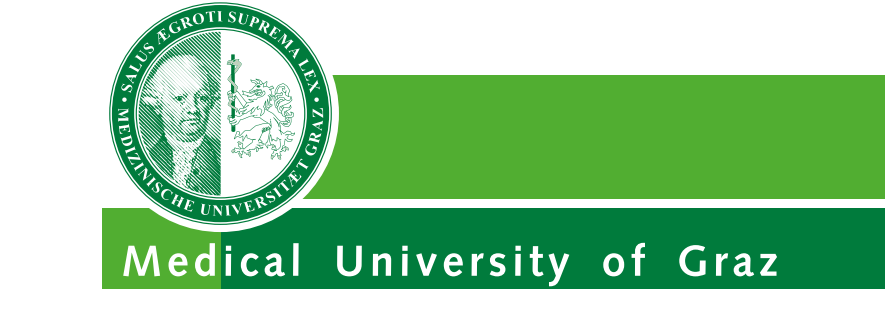

${ }^{2}$ Medical University of Graz

Division of Plastic, Esthetic and Reconstructive Surgery

Graz, Austria

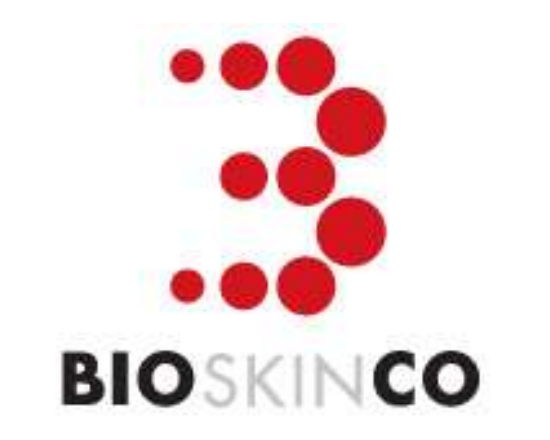

BIOCELLULAR SCIENCE

${ }^{3}$ Bioskinco GmbH Würzburg, Germany

\section{Introduction}

The initial phase of the local inflammatory response after burn injuries may be predictive for the subsequent wound healing process. Further, knowledge about the local inflammatory response may support physicians and care attendants to select optimal treatments for enhanced wound healing. However, little is known about the local inflammatory response in the damaged skin due to the lack of adequate sampling methods.

Thus, we aimed to optimize the sampling method dermal open flow microperfusion (dOFM) for the continuous sampling of interstitial fluid (ISF) directly from the burned skin to enable monitoring of the local inflammatory response. We performed a feasibility study in freshly excised human skin with burn injuries induced by two different stimuli.

\section{Materials and Methods}

In this feasibility study three test areas were applied on freshly excised human skin (Fig. 1). One test area was treated with a scald stimulus induced by boiling water $\left(100^{\circ} \mathrm{C}, 10\right.$ seconds), the second with a burn stimulus induced by a heated metal block $\left(100^{\circ} \mathrm{C}, 10\right.$ seconds). The third test area was left untreated to serve as negative control site. Three OFM probes were inserted into each test area at a depth of about $0.8 \mathrm{~mm}$. The temperature in the dermis was measured with temperature sensors during each stimulus.

ISF was continuously sampled with dOFM directly from the dermis. Sampling started half an hour before the respective stimulus and was performed for 48 hours after application of the respective stimulus. This study was performed in a climate chamber to regulate the temperature to $32^{\circ} \mathrm{C} \pm 2^{\circ} \mathrm{C}$ (skin surface temperature) and the humidity to 40 - $60 \%$.

The halfhourly and hourly samples were used to screen for approximately 100 inflammatory biomarkers (OLINK Proteomics, Targeted protein biomarker discovery). Punch biopsies (B in Fig. 1) were taken one hour after application of the stimulus and at the end of the experiment (48 hours post-burn) for histological verification of the burn injuries.

\section{Results and Discussion}

Cytokine levels differed between the treated areas and the control site (e.g. VEGF-A, Fig. 2) during the whole monitoring period of 48 hours. The areas treated with burn and scalding stimulus, respectively showed comparable cytokine levels.

The heated metal block induced a higher temperature in the skin than the scalding stimulus (Fig. 3).

- Histological evaluations revealed detached epidermis and blisters 48hours post-burn (Fig. 4) which indicate a 2nd degree burn.

\section{Conclusion and Outlook}

This feasibility study shows the successful application of dOFM as an adequate sampling method to examine the local inflammatory response to two different topically applied burn stimuli in this feasibility study. Further studies in excised human skin will be performed to verify these results.

Our future vision is to generate data from preclinical and clinical in vivo studies in burned patients that may enable the discovery of local cytokine profiles to predict the individual progress of wound healing and to promote the development of specific wound dressings for individual therapy.

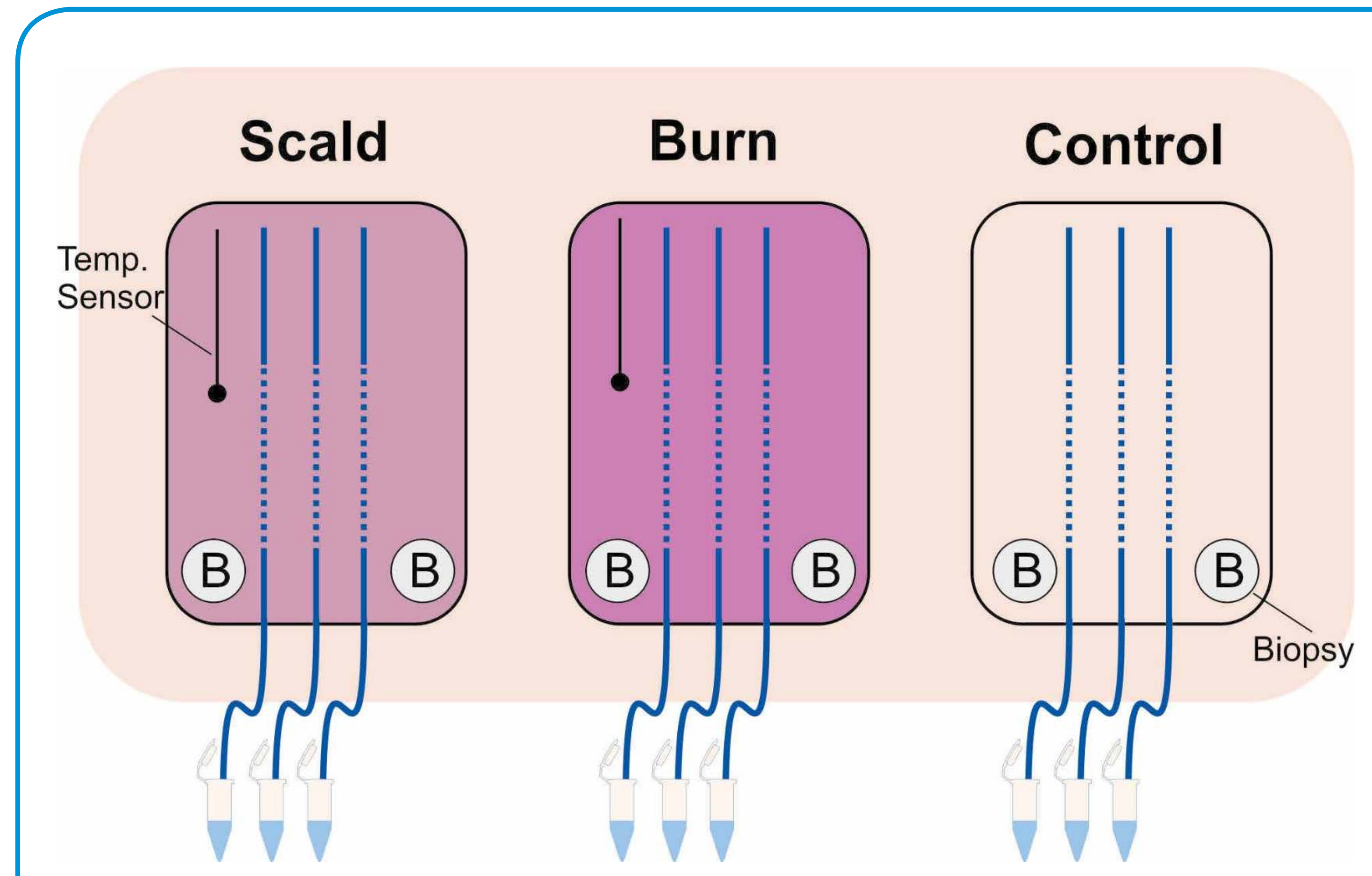

Figure 1: Scheme of the experimental design: In each test area three dOFM probes were inserted to sample ISF directly from the dermis (Scald. burn stimulus induced by boiling water, Burn. burn stimulus induced by a heated metal block and Control. nostimulus)

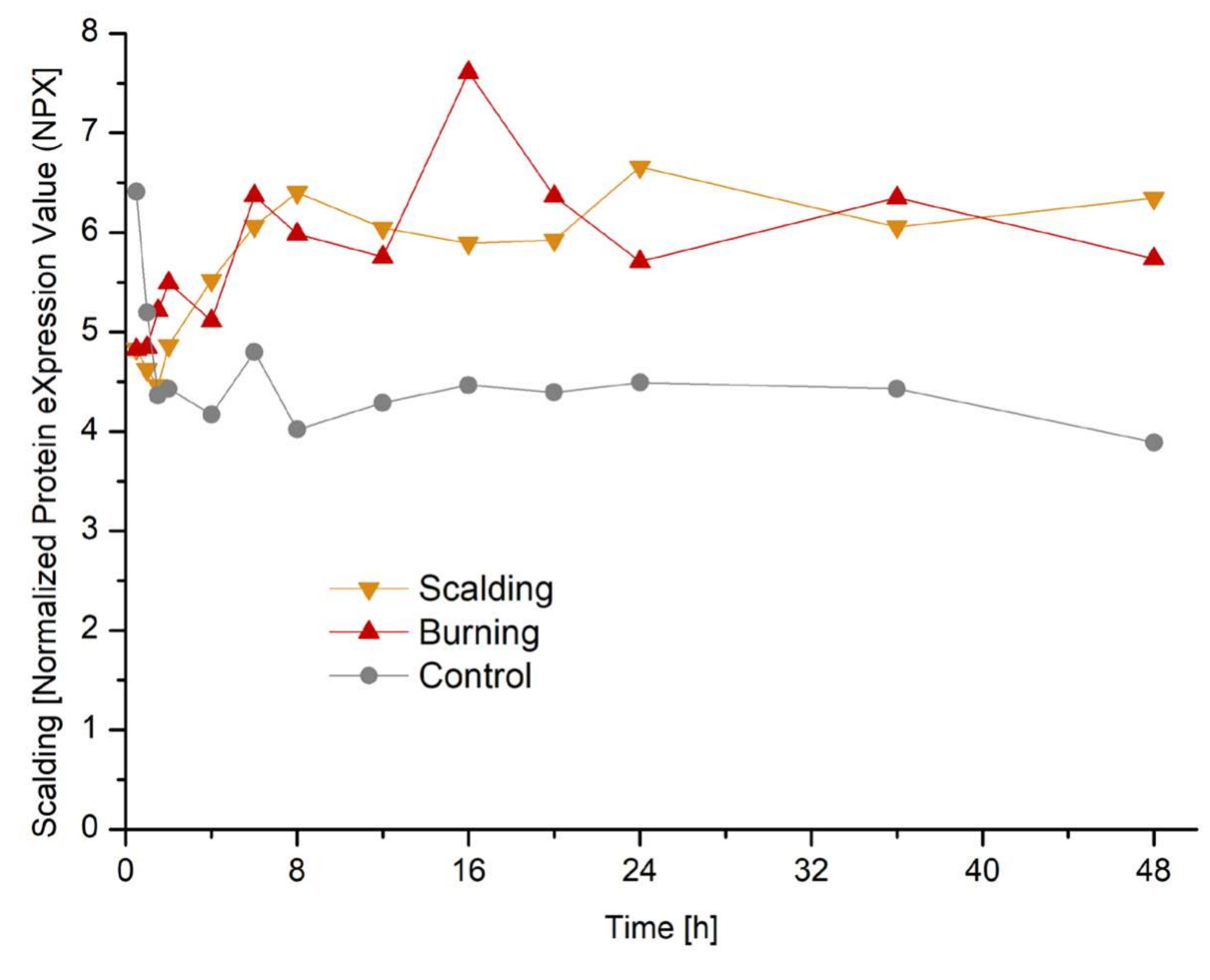

Figure 2: Concentration-time curves for VEFG-A measured in the ISF of the three test areas (Scalding: burn stimulus induced by boiling water Burning: burn stimulus induced by a heated metal block and Control: no stimulus)

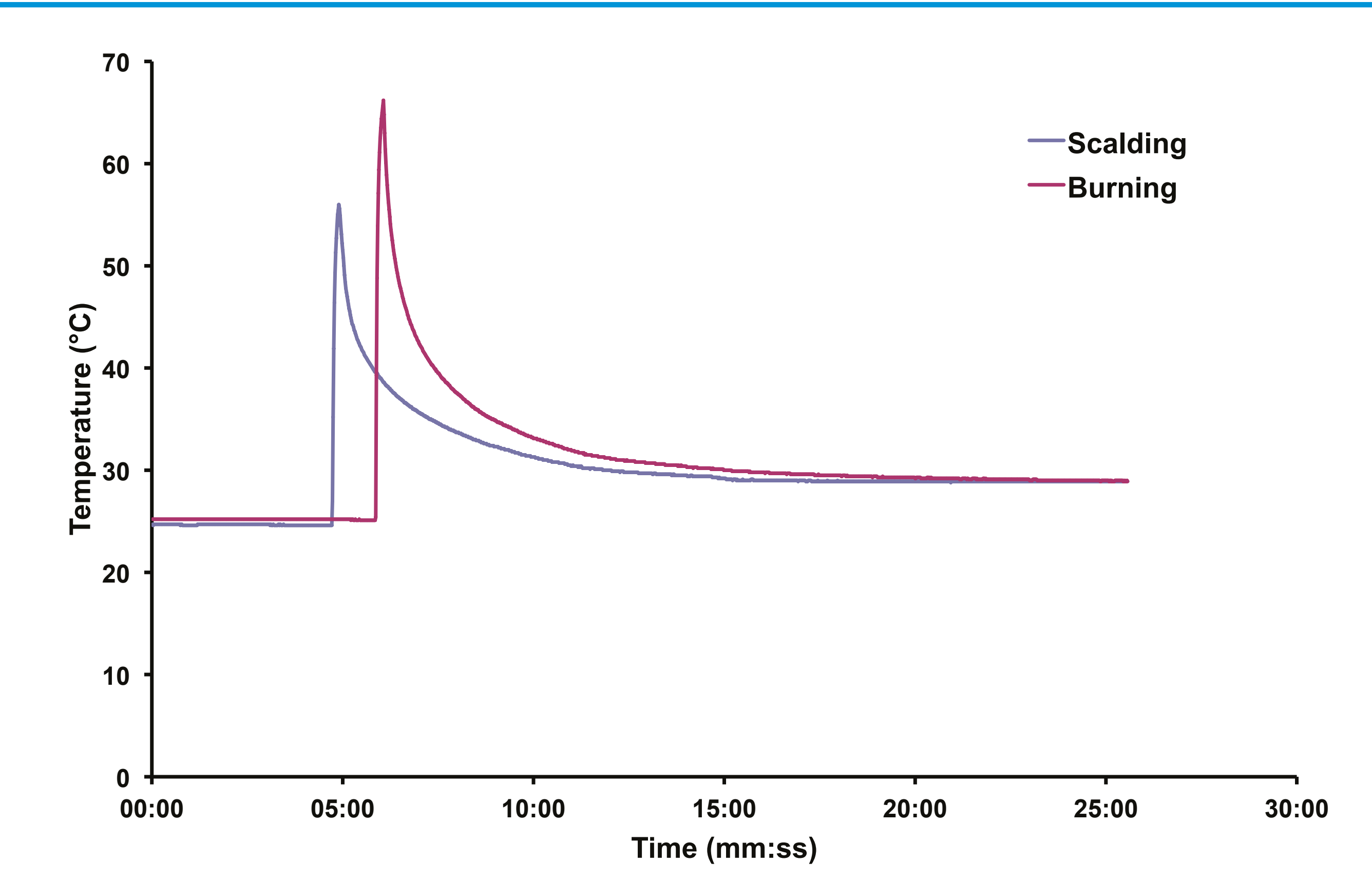

Figure 3: Temperature-time curves for the two burning stimuli. (Scalding: burn stimulus induced by boiling water, Burning: burn stimulus induced by a heated metal block)

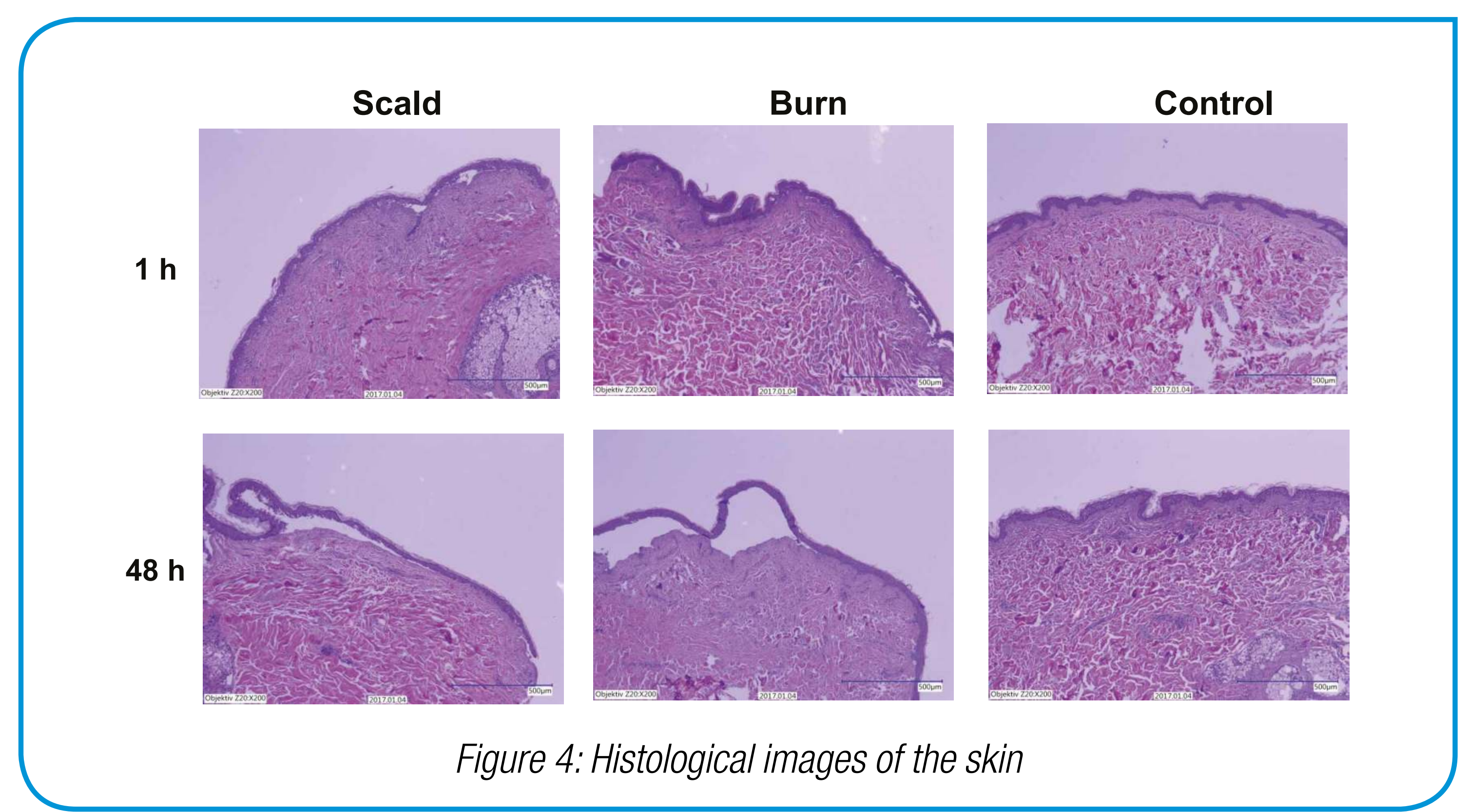

